\title{
STABILITY CROSSING CURVES OF LINEAR SYSTEMS WITH SHIFTED FRACTIONAL $\gamma$-DISTRIBUTED DELAYS
}

\author{
Irinel Constantin Morărescu* \\ André Ricardo Fioravanti** \\ Silviu-Iulian Niculescu ${ }^{* * *}$ Catherine Bonnet ${ }^{* *}$ \\ * Joseph Fourier University, Jean Kuntzmann Laboratory \\ Tour IRMA, 51 rue des Mathématiques, 38400 Saint \\ Martin d'Hères, France. \\ constantin.morarescu@imag.fr \\ ** INRIA Paris-Rocquencourt, Domaine de Voluceau \\ B.P. 105, 78153, Le Chesnay Cedex, France. \\ \{Andre.Fioravanti, Catherine.Bonnet\}@inria.fr \\ *** L2S (UMR CNRS 8506), CNRS-Supélec, \\ 3, rue Joliot Curie, 91192, Gif-sur-Yvette, France. \\ Silviu.Niculescu@lss.supelec.fr
}

\begin{abstract}
This paper focuses on the characterization of the stability crossing curves of a class of linear systems with shifted fractional gamma-distributed delay. First, we describe the frequency crossing set, i.e., the set of frequencies where the characteristic roots may cross the imaginary axis as the parameters change. Next, we describe the stability crossing curves, i.e., the set of parameters (average delay, gap) such that there exists at least one characteristic root on the imaginary axis. Such stability crossing curves divide the parameter-space into different regions, such that within each such region, the number of strictly unstable roots is fixed. The classification of the stability crossing curves is also discussed and illustrated. Copyright (C) IFAC 2009
\end{abstract}

Keywords: distributed delay; $\gamma$-kernels; stability crossing curves.

\section{INTRODUCTION}

One of the classical problems in the stability analysis of a dynamical system is to understand how a stable equilibrium state or a steady motion become stable/unstable with a change of the parameters, see, e.g. Seyranian and Maylibaev [2003]. Without any loss of generality, the corresponding parameter-space will be divided in various stability and instability domains, such that, in the linear case, the number of unstable characteristic roots is invariant with respect to all the points of the parameter space inside the region, and such that for each point of the boundaries the corresponding characteristic equation has at least one root on the imaginary axis. In the sequel, such a root will be called critical, and corresponding frequency a crossing frequency. It is well known that such a problem becomes particularly difficult in the delay systems case, see, for instance, El'sgolts and Norkin [1973], Niculescu [2001], Gu et al. [2003] and the references therein. Among the methods to perform such a task, we cite in particular two "dual" methods: the $D$ subdivision and $\tau$-decomposition, respectively. As 
explained in Michiels and Niculescu [2007], the distinction between these methods lies on the way the delay is treated.

The current paper focuses on the characterization of stability crossing curves for a class of systems of linear systems with shifted fractional gammadistributed delay. Such kernels have been used in different applicative areas since they offer an excellent fit to the data in many situations as, for example in biology, or networks control. More precisely, the kernel may represent the distribution of maturation delay of hematopoïetic stem cells as in Bernard et al. [2001], Haurie et al. [2000], Hearn et al. [1998], or the total network delay where the gamma distribution is used to model queueing delay as discussed in Li and Mason [2007].

In the sequel, we will consider the parameter space defined by the delay terms, that is (average delay, gap) and the proposed approach follows the lines of Gu et al. [2005], Morărescu et al. [2007] for the class of systems under consideration. Without any lack of generality, the approach we are proposing enters in the class of $\tau$-decomposition methods, and it can be resumed as follows: we start by describing the frequency crossing set. For this class of systems, we characterize the stability crossing curves, i.e., the set of parameters (average delay, gap) such that there is at least one pair of characteristic roots on the imaginary axis. Such stability crossing curves divide the parameter space $\mathbb{R}_{+}^{2}$ into different regions, such that within each such region, the number of strictly unstable characteristic roots is fixed. The classification of the stability crossing curves is largely discussed. It is worth to mention that to the best of the authors' knowledge, there does not exist similar results in the open literature for the class of fractional systems considered here.

The following standard notation will be adopted: $\mathbb{C}\left(\mathbb{C}^{+}, \mathbb{C}^{-}\right)$is the set of complex numbers (with strictly positive, and strictly negative real parts), and $j=\sqrt{-1}$. For $z \in \mathbb{C}, \angle z, \Re(z)$ and $\Im(z)$ define the argument, the real part and the imaginary part of $z . \mathbb{R}\left(\mathbb{R}^{+}, \mathbb{R}^{-}\right)$denotes the set of real numbers (larger or equal to zero, smaller or equal to zero). $\mathbb{N}$ is the set of natural numbers, including zero and $\mathbb{Z}$ the set of integers.

\section{PRELIMINARIES}

Consider the problem of stability analysis of a general class of delay differential equations (DDE) that can be described in frequency-domain by the following characteristic function:

$$
H(s, \alpha, \beta)=Q(s)+P(s) f(s, \alpha, \beta),
$$

where $P$ and $Q$ denote polynomials with $\operatorname{deg}(P)<$ $\operatorname{deg}(Q), f$ is an analytic function (in a domain which contains the right half-plane), containing at least one exponential function such that (1) is transcendental, with $\alpha, \beta$ real parameters controlling the behavior of the system. Furthermore, we assume that:

Assumption 1. The polynomials $P(s)$ and $Q(s)$ do not have common zeros.

It is obvious that if Assumption 1 is violated then $P, Q$ have a common factor $c(s) \neq$ constant. Simplifying by $c(s)$ we get a system described by (1) satisfying Assumption 1.

Let us precise that, for instance, a complete characterization of $H_{\infty}$-stability for such general systems is not known. So that, 'stability' word is used as an abuse of language in this section as well as in section III, describing only the absence of poles in the closed right half-plane. However, for systems considered in section IV, it can be deduced from Bonnet and Partington [2002] that $H_{\infty}$-stability is equivalent to the condition 'no poles in the right half-plane' and 'stability' has a stronger meaning there.

As mentioned in the Introduction, the aim of this paper is to present the way the location of the roots of the characteristic function of the type (1), behaves in the parameter-space defined by the pair $(\alpha, \beta)$. Without any loss of generality, the continuity dependence of the roots of the characteristic function with respect to the parameters $\alpha$ and $\beta^{1}$ reduce the (stability) analysis to the following problems:

(a) first, to detect crossings with respect to the imaginary axis $j \mathbb{R}$ since such crossing are related to changes in the stability behavior. In other words, we need to compute the frequency crossing set denoted by $\Omega$, which consists of all positive frequencies corresponding to the existence of at least one critical characteristic root. As we shall see later, the frequency crossing set is reduced to a finite collection of intervals. This set will be derived by using geometric arguments; (b) second, to describe the behavior of critical roots under changes of parameters in $(\alpha, \beta)$ parameter space. More precisely, we will detect switches (reversals) corresponding to the situation when critical characteristic roots cross $j \mathbb{R}$ towards instability (stability). Excepting some explicit computation of the crossing direction, we will also briefly discuss the smoothness properties as well as with some appropriate classification of the stability crossing boundaries.

1 See, for instance, Datko [1978] or El'sgolts and Norkin [1973] for further remarks on the dependence of the roots of DDE's with respect to delay parameters, see also Bonnet and Partington [2007] for some considerations in the fractional case 
Finally, another useful related concept is represented by the characteristic crossing curves consisting of all pairs $(\alpha, \beta)$ for which there exists at least one value $\omega \in \Omega$ such that $H(j \omega, \alpha, \beta)=0$.

\section{SMOOTHNESS OF THE CROSSING CURVES AND CROSSING DIRECTION}

In the sequel, let us consider that the frequency crossing set $\Omega$ is given and the stability crossing curves are described by the smooth mappings $\omega \mapsto \alpha(\omega), \omega \mapsto \beta(\omega)$. Denote also by $\mathcal{T}_{h}$ an arbitrary crossing curve and consider the following decompositions into real/imaginary parts:

$$
\begin{aligned}
& R_{0}+j I_{0}=\left.j \frac{\partial H(s, \alpha, \beta)}{\partial s}\right|_{s=j \omega}, \\
& R_{1}+j I_{1}=\left.\frac{\partial H(s, \alpha, \beta)}{\partial \alpha}\right|_{s=j \omega}, \\
& R_{2}+j I_{2}=\left.\frac{\partial H(s, \alpha, \beta)}{\partial \beta}\right|_{s=j \omega} .
\end{aligned}
$$

Then, since $H(s, \alpha, \beta)$ is an analytic function of $s, \alpha$ and $\beta$, the implicit function theorem indicates that the tangent of $\mathcal{T}_{h}$ can be expressed as

$$
\begin{array}{r}
\left(\begin{array}{c}
\frac{\mathrm{d} \alpha}{\mathrm{d} \omega} \\
\frac{\mathrm{d} \beta}{\mathrm{d} \omega}
\end{array}\right)_{s=j \omega}=\left(\begin{array}{cc}
R_{1} & R_{2} \\
I_{1} & I_{2}
\end{array}\right)^{-1}\left(\begin{array}{c}
R_{0} \\
I_{0}
\end{array}\right) \\
=\frac{1}{R_{1} I_{2}-R_{2} I_{1}}\left(\begin{array}{c}
R_{0} I_{2}-R_{2} I_{0} \\
R_{1} I_{0}-R_{0} I_{1}
\end{array}\right),
\end{array}
$$

provided that

$$
R_{1} I_{2}-R_{2} I_{1} \neq 0 .
$$

It follows that $\mathcal{T}_{h}$ is smooth everywhere except possibly at the points where either (3) is not satisfied, or when

$$
\frac{\mathrm{d} \alpha}{\mathrm{d} \omega}=\frac{\mathrm{d} \beta}{\mathrm{d} \omega}=0
$$

If (4) is satisfied then straightforward computations show us that $R_{0}=I_{0}=0$. In other words $s=j \omega$ is a multiple solution of (1).

We will call the direction of the curve that corresponds to increasing $\omega$ the positive direction. We will also call the region on the left hand side as we head in the positive direction of the curve the region on the left. To establish the direction of crossing we need to consider $\alpha$ and $\beta$ as functions of $s=\sigma+j \omega$, i.e., functions of two real variables $\sigma$ and $\omega$, and partial derivative notation needs to be adopted. Since the tangent of $\mathcal{T}_{h}$ along the positive direction is $\left(\frac{\partial \alpha}{\partial \omega}, \frac{\partial \beta}{\partial \omega}\right)$, the normal to $\mathcal{T}_{h}$ pointing to the left hand side of positive direction is $\left(-\frac{\partial \beta}{\partial \omega}, \frac{\partial \alpha}{\partial \omega}\right)$. Corresponding to a pair of complex conjugate solutions crossing the imaginary axis along the horizontal direction, $(\alpha, \beta)$ moves along the direction $\left(\frac{\partial \alpha}{\partial \sigma}, \frac{\partial \beta}{\partial \sigma}\right)$. So, as $(\alpha, \beta)$ crosses the stability crossing curves from the right hand side to the left hand side, a pair of complex conjugate solutions crosses the imaginary axis to the right half plane, if

$$
\left(\frac{\partial \alpha}{\partial \omega} \frac{\partial \beta}{\partial \sigma}-\frac{\partial \beta}{\partial \omega} \frac{\partial \alpha}{\partial \sigma}\right)_{s=j \omega}>0,
$$

i.e. the region on the left of $\mathcal{T}_{h}$ gains two solutions on the right half plane. If the inequality (5) is reversed then the region on the left of $\mathcal{T}_{h}$ loses two right half plane solutions. Similar to (3) we can express

$$
\begin{aligned}
\left(\begin{array}{c}
\frac{\partial \alpha}{\partial \sigma} \\
\frac{\partial \beta}{\partial \sigma}
\end{array}\right)_{s=j \omega} & =\left(\begin{array}{cc}
R_{1} & R_{2} \\
I_{1} & I_{2}
\end{array}\right)^{-1}\left(\begin{array}{c}
I_{0} \\
-R_{0}
\end{array}\right) \\
& =\frac{1}{R_{1} I_{2}-R_{2} I_{1}}\left(\begin{array}{c}
R_{0} R_{2}+I_{0} I_{2} \\
-R_{0} R_{1}-I_{0} I_{1}
\end{array}\right) .
\end{aligned}
$$

Proposition 1. Assume $\omega \in \Omega$, and $s=j \omega$ is a simple root of (1) and $H\left(j \omega^{\prime}, \alpha, \beta\right) \neq 0, \forall \omega^{\prime}>$ $0, \omega^{\prime} \neq \omega$ (i.e. $(\alpha, \beta)$ is not an intersection point of two curves or different sections of a single curve). Then as $(\alpha, \beta)$ moves from the region on the right to the region on the left of the corresponding crossing curve, a pair of solutions of (1) crosses the imaginary axis to the right (through $s= \pm j \omega$ ) if $R_{1} I_{2}-R_{2} I_{1}>0$. The crossing is to the left if the inequality is reversed.

\section{STABILITY CROSSING CURVES CHARACTERIZATION}

In the sequel let us consider

$$
f(s, \alpha, \beta)=\frac{1}{(s+\alpha)^{m / n}} e^{-s \beta}
$$

where $m, n \in \mathbb{Z}, n \neq 0, \alpha \in \mathbb{R}^{+}$and $s \in \mathbb{C} \backslash$ $(-\infty,-\alpha]$. In order to deal with fractional delay systems of retarded type, we make the following assumption.

Assumption 2. $\operatorname{deg}(P)-\frac{m}{n}<\operatorname{deg}(Q)$.

In this case, the characteristic function (1) rewrites as:

$$
H(s, \alpha, \beta)=Q(s)+P(s) \frac{1}{(s+\alpha)^{m / n}} e^{-s \beta} .
$$

The aim of this section is to characterize the stability crossing curves in the $(\alpha, \beta)$ parameter space. The presentation is as simple as possible, and intuitive. 


\subsection{Identification of the crossing set}

Let $\mathcal{T}$ denote the set of all $(\alpha, \beta) \in \mathbb{R}_{+} \times \mathbb{R}_{+}$ such that $(7)$ has at least one zero on $j \mathbb{R}$. Any $(\alpha, \beta) \in \mathcal{T}$ is known as a crossing point. The set $\mathcal{T}$, which is the collection of all crossing points, is known as the stability crossing curves. If $\omega \in$ $\mathbb{R}$ and $(\alpha, \beta) \in \mathbb{R}_{+} \times \mathbb{R}_{+}$then $H(j \omega, \alpha, \beta)=$ $\overline{H(-j \omega, \alpha, \beta)}$. Therefore, in the sequel, we only need to consider positive $\omega$.

Proposition 2. The frequency crossing set $\Omega$ consists of a finite union of bounded intervals.

PROOF. Let $\omega \in \Omega$ be a crossing frequency. Considering $s=j \omega$ and applying the modulus to (7) one gets

$$
|Q(j \omega)|=\left|\frac{P(j \omega)}{(j \omega+\alpha)^{m / n}}\right| .
$$

If $Q(j \omega)=0$ one can easily derive that $P(j \omega)=0$ and Assumption 1 is violated. In a similar way $P(j \omega)=0$ implies $Q(j \omega)=0$. Therefore, $Q(j \omega) P(j \omega) \neq 0, \forall \omega \in \Omega$ and (8) rewrites as

$$
\left|\frac{P(j \omega)}{Q(j \omega)(j \omega+\alpha)^{m / n}}\right|=1 .
$$

Since $\operatorname{deg}(P)<\operatorname{deg}(Q)+\frac{m}{n}$ the left hand side of (9) tends to 0 when $\omega \mapsto \infty$. This means that there exists $\omega_{M}>0$ such that $\Omega \cap \mathbb{R}_{+} \subset\left(0, \omega_{M}\right]$. Furthermore (9) has solutions $(\omega, \alpha(\omega))$ if and only if

$$
\left\{\begin{array}{l}
|\omega|^{m / n} \leq\left|\frac{P(j \omega)}{Q(j \omega)}\right|, \quad m / n>0 \\
|\omega|^{-m / n} \leq\left|\frac{Q(j \omega)}{P(j \omega)}\right|, \quad m / n<0
\end{array}\right.
$$

In this case

$$
\alpha^{2}=\left|\frac{P(j \omega)}{Q(j \omega)}\right|^{2 n / m}-\omega^{2} .
$$

It is clear that (10) holds for $\omega$ in a finite union of intervals and the proof is completed.

\subsection{Identification of crossing points}

Proposition 3. Given $\omega \in \Omega \cap \mathbb{R}_{+}$, the corresponding crossing points are given by:

$$
\begin{aligned}
\alpha & =\sqrt{\left|\frac{P(j \omega)}{Q(j \omega)}\right|^{2 n / m}-\omega^{2}}, \\
\beta & =\frac{1}{\omega}((2 p+1) \pi+\angle P(j \omega)-\angle Q(j \omega) \\
& \left.-\frac{m}{n} \arctan \left(\frac{\omega}{\alpha}\right)\right)
\end{aligned}
$$

where $p \in \mathbb{Z}$.
PROOF. The definition of $\alpha$ follows directly from (11). The definition of $\beta$ is obtained from (7) since $\angle Q(j \omega)$ must be equal modulo an integer number of $2 \pi$ with $\angle\left(\frac{P(j \omega)}{(j \omega+\alpha)^{m / n}} e^{-j \omega \beta}\right)$.

In the sequel we consider $\Omega=\bigcup_{k=1}^{N} \Omega_{k}$ and we do not restrict $\angle Q(j \omega)$ and $\angle P(j \omega)$ to a $2 \pi$ range. Rather we allow them to vary continuously within each interval $\Omega_{k}$. Thus, for each fixed $p \in \mathbb{Z},(12)$ and (13) define a continuous curve. Let us denote by $\mathcal{T}_{k, \pm}^{p}$ the curve corresponding to $\Omega_{k}$ with $p \in \mathbb{Z}$ and $\alpha$ defined using "+" or "-", respectively. We note that we have an infinite number of curves corresponding to each interval.

\subsection{Classification of the crossing curves}

Let the left and right end points of interval $\Omega_{k}$ be denoted as $\omega_{k}^{\ell}$ and $\omega_{k}^{r}$, respectively. Due to Assumptions 1 and 2, it is not difficult to see that each end point $\omega_{k}^{\ell}$ or $\omega_{k}^{r}$ must belong to one, and only one, of the following three types:

Type 1a. It satisfies the equation $Q(j \omega)=0$ and $m / n>0$.

Type 1b. It satisfies the equation $P(j \omega)=0$ and $m / n<0$.

Type 2. It satisfies the equation

$$
|\omega|^{m / n}=\left|\frac{P(j \omega)}{Q(j \omega)}\right| .
$$

Type 3. It equals 0 .

Let us denote a generic end-point by $\omega_{0}$, which may be either a left end or a right end of an interval $\Omega_{k}$. Then the corresponding points in $\mathcal{T}_{k,+}^{p}$ may be described as follows:

If $\omega_{0}$ is of type $\mathbf{1 a}$, then as $\omega \rightarrow \omega_{0} \alpha \rightarrow \infty$ and

$$
\beta \rightarrow \frac{1}{\omega_{0}}\left((2 p+1) \pi+\angle P\left(j \omega_{0}\right)-\lim _{\omega \rightarrow \omega_{0}} \angle Q(j \omega)\right) \text {. }
$$

Obviously,

$$
\lim _{\omega \rightarrow \omega_{0}} \angle Q(j \omega)=\angle\left[\frac{d}{d \omega} Q(j \omega)\right]_{\omega \rightarrow \omega_{0}}
$$

if $\omega_{0}$ is the left end point $\omega_{k}^{\ell}$ of $\Omega_{k}$, and

$$
\lim _{\omega \rightarrow \omega_{0}} \angle Q(j \omega)=\angle\left[\frac{d}{d \omega} Q(j \omega)\right]_{\omega \rightarrow \omega_{0}}+\pi
$$

if $\omega_{0}$ is the right end point $\omega_{k}^{r}$ of $\Omega_{k}$. In other words, $\mathcal{T}_{k,+}^{p}$ approaches a horizontal line.

If $\omega_{0}$ is of type $\mathbf{1 b}$, then as $\omega \rightarrow \omega_{0} \alpha \rightarrow \infty$ and

$$
\beta \rightarrow \frac{1}{\omega_{0}}\left((2 p+1) \pi-\angle Q\left(j \omega_{0}\right)+\lim _{\omega \rightarrow \omega_{0}} \angle P(j \omega)\right) .
$$

Again $\mathcal{T}_{k,+}^{p}$ approaches a horizontal line.

If $\omega_{0}$ is of type $\mathbf{2}$, then $\alpha=0$. In other words, $\mathcal{T}_{k}^{m}$ intersects the $\tau$-axis at $\omega=\omega_{0}$. 


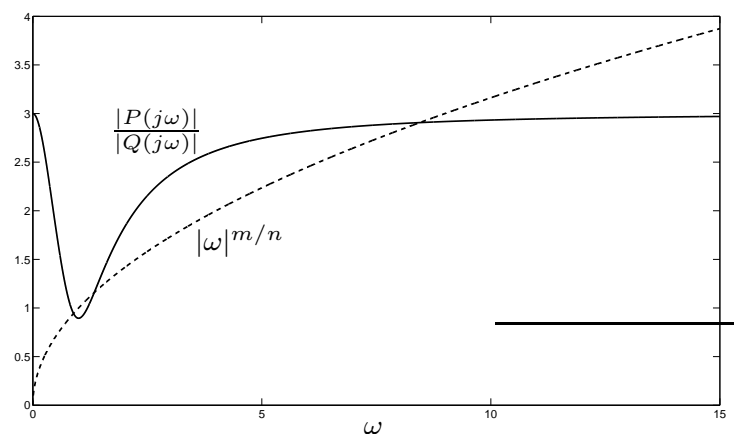

Fig. 1. Example 1: $\frac{|P(j \omega)|}{|Q(j \omega)|}$ and $|\omega|^{m / n}$ against $\omega$.

Obviously, only $\omega_{1}^{\ell}$ may be of type 3 . In this case, $\alpha \rightarrow|P(0) / Q(0)|^{n / m}$ and $\beta \rightarrow \infty$ as $\omega \rightarrow 0$. Therefore $(\alpha, \beta)$ approaches a vertical line. It is noteworthy that in some particular cases for $p=0$ one gets $\pi+\angle P(j \omega)-\angle Q(j \omega)=0$ implying:

$$
\begin{aligned}
& \beta \underset{\omega \rightarrow 0}{\longrightarrow}\left[\frac{d}{d \omega} \angle P(j \omega)\right]_{\omega \rightarrow 0}-\left[\frac{d}{d \omega} \angle Q(j \omega)\right]_{\omega \rightarrow 0} \\
& -\frac{m}{n}\left|\frac{P(0)}{Q(0)}\right|^{-n / m}
\end{aligned}
$$

that is

$$
\beta \underset{\omega \rightarrow 0}{\longrightarrow} \frac{P^{\prime}(0)}{P(0)}-\frac{Q^{\prime}(0)}{Q(0)}-\frac{m}{n}\left|\frac{P(0)}{Q(0)}\right|^{-n / m},
$$

resulting in $(\alpha, \beta)$ approaching a limit point.

Moreover, we can see from (7) that whenever there exists $\alpha \in \mathbb{R}^{+}$such that $\alpha^{m / n}=-P(0) / Q(0)$, then $\omega=0$ will be a solution of the characteristic equation independently of the value of $\beta$ so that $\mathcal{T}_{k,+}^{p}$ includes a vertical line.

We say an interval $\Omega_{k}$ is of type $\ell r$ if its left end is of type $\ell$ and its right end is of type $r$. We may accordingly divide these intervals into 10 types.

\section{EXAMPLES}

In order to illustrate the proposed results, we shall consider two academic examples:

Example 1. (types 32 and 22). Let $m=1, n=2$, $P(s)=3 s^{2}+2 s+3$ and $Q(s)=s^{2}+s-1$. Figure 1 plots $|P(j \omega)| /|Q(j \omega)|$ and $|\omega|^{m / n}$ against $\omega$. From the plot, it can be seen that the crossing set $\Omega$ contains two intervals, $\Omega_{1}=[0,0.8895]$ of type 32 and $\Omega_{2}=[1.34,8.4489]$ of type 22 . Figure 2 shows the stability crossing curves for $\Omega_{1}$. With $\omega \rightarrow 0$, we can notice that for $p=0$, the curve is in the special case where $(\alpha, \beta)$ goes first to a limit point and then join a vertical axis. But for all other cases, the graphic is asymptotic to a vertical line. Finally, when $\omega \rightarrow \omega_{1}^{r}=0.8895$, all curves end on the $\beta$-axis. Figure 3 shows the

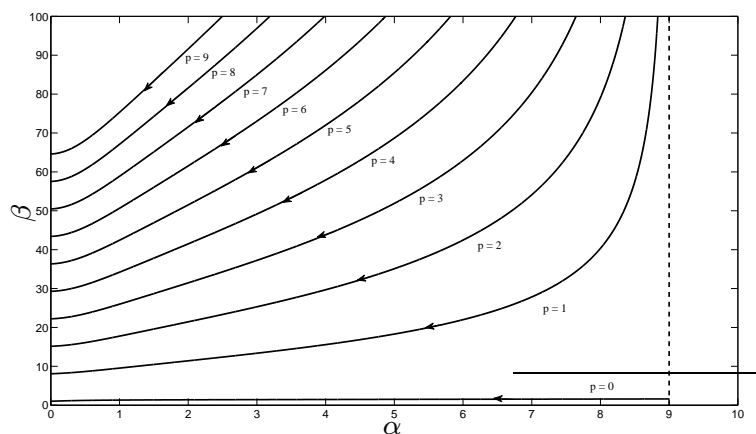

Fig. 2. Example 1: $\mathcal{T}_{1,+}^{p}$ for $p=0, \ldots, 9$ (type 32).

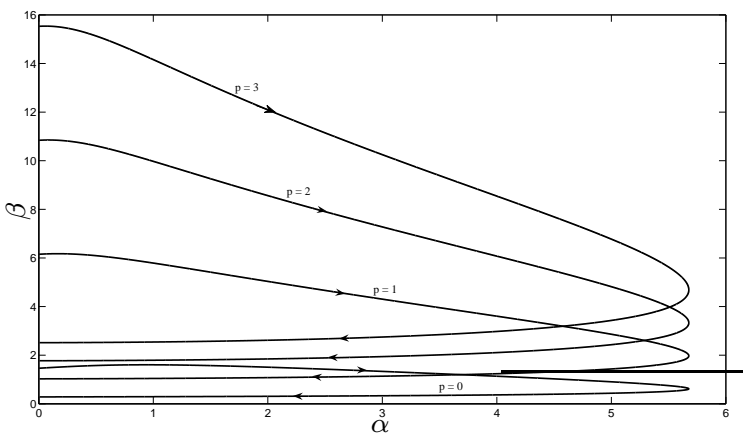

Fig. 3. Example 1: $\mathcal{T}_{2,+}^{p}$ for $p=0,1,2,3$ (type 22).

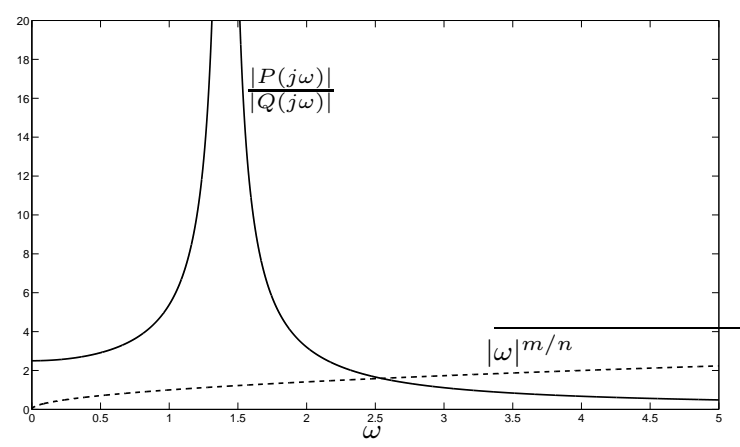

Fig. 4. Example 2: $\frac{|P(j \omega)|}{|Q(j \omega)|}$ and $|\omega|^{m / n}$ against $\omega$.

stability crossing curves for $\Omega_{2}$, which is of type 22 . As it can be easily remarked, it consists of a series of curves with both ends on the $\beta$-axis.

Example 2. (types 31a and 1a2). For the second example, let us consider $m=1, n=2$, $P(s)=2 s+5$ and $Q(s)=s^{2}+2$. Figure 4 plots $|P(j \omega)| /|Q(j \omega)|$ and $|\omega|^{m / n}$ against $\omega$. From the plot, it can be seen that the crossing set $\Omega$ contains two intervals, $\Omega_{1}=[0, \sqrt{2}]$ of type $31 \mathrm{a}$ and $\Omega_{2}=[\sqrt{2}, 2.5441]$ of type $1 \mathrm{a} 2$. Figure 5 shows the stability crossing curves for $\Omega_{1}$. We precise that as $\omega \rightarrow 0$ the graphic asymptotically approaches the vertical line $\alpha=6.25$. When $\omega \rightarrow \omega_{1}^{r}=\sqrt{2}$, all the curves are asymptotic to a horizontal line. To conclude the academic examples, Figure 6 shows the stability crossing curves for $\Omega_{2}$, which is of type 1a2, and consists of a series of curves which starts on a horizontal line and ends on the $\beta$ axis. It can be shown that this system is stable 


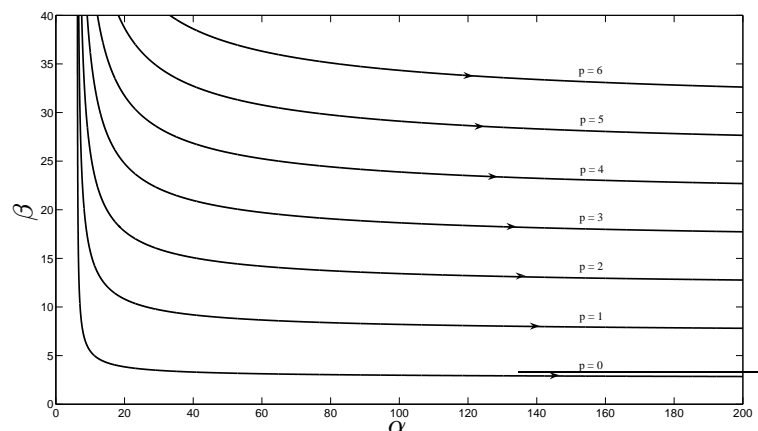

Fig. 5. Example 2: $\mathcal{T}_{1,+}^{p}$ for $p=0, \ldots, 6$ (type 31a).

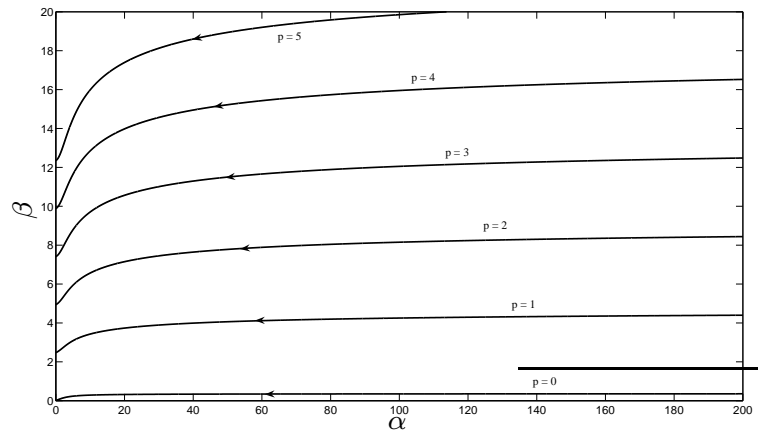

Fig. 6. Example 2: $\mathcal{T}_{2,+}^{p}$ for $p=0, \ldots, 5$ (type 1a2). for $(\alpha, \beta)=(0,0)$, but there is no value of $\alpha$ for which the system does not cross the stability curves when $\beta$ increases.

\section{CONCLUDING REMARKS}

This paper addressed the asymptotic stability of a class of linear systems with fractional $\gamma$ distributed delays. More precisely, the characterizations of the stability crossing curves and corresponding crossing frequency set are proposed.

\section{ACKNOWLEDGEMENTS}

The work of I.C. MORĂRESCU was partially funded by the Romanian project "IDEI" CNCSIS ID 548: "Development of models and methods for the identification and control of biotechnological and biological systems."

\section{REFERENCES}

Bernard S., Bélair J. and Mackey M.C., (2001). Sufficient conditions for stability of linear differential equations with distributed delay, Discrete and continuous dynamical systems - Series B, vol. 1, pp. 233-256.

Bonnet C. and Partington J.R. (2002). Analysis of fractional delay systems of retarded and neutral type, Automatica, vol. 38, 1133-1138.
Bonnet C. and Partington J.R. (2007). Stabilization of some fractional delay systems of neutral type, vol. 43, 2047-2053.

Datko, R. (1978). A procedure for determination of the exponential stability of certain differential-difference equations. Quart. Appl. Math., vol. 36, 279-292.

El'sgolts', L.E. and Norkin, S.B. (1973). Introduction to the theory and applications of differential equations with deviating arguments Academic Press: New York.

Gu, K., Kharitonov, V.L. and Chen, J. (2003). Stability of time-delay systems (Birkhauser: Boston).

Gu, K., Niculescu, S.-I., and Chen, J. (2005). On stability of crossing curves for general systems with two delays, J. Math. Anal. Appl., vol. 311, 231-253.

Haurie C., Dale D.C., Rudnicki R., and Mackey M.C. (2000). Modeling complex neutrophil dynamics in the grey collie, J. theor. Biol., 204, 505-519.

Hearn T., Haurie C. and Mackey M.C. (1998). Cyclical neutropenia and the peripheral control of white blood cell production, J. Theor. Biology, vol. 192, 226-232.

Li, H., Mason, L (2007). Synthesis of networks delays for voice packets in Service Overlay Networks, QSHINE'07, August 14-17 2007, Vancouver, Canada.

Michiels, W. and Niculescu, S.-I. (2007). Stability and stabilization of time-delay systems. An eigenvalue-based approach, SIAM: Philadelphia.

Morărescu, C.I. (2006). Qualitative analysis of distributed delay systems: Methodology and algorithms (Ph.D. thesis, University of Bucharest/Université de Technologie de Compiègne, September 2006).

Morărescu, C.-I., Niculescu, S.-I. and Gu, K. (2007). Stability crossing curves of shifted gamma-distributed delay systems. SIAM Journal on Dynamical Systems, vol. 6, 475-493.

Niculescu, S. -I. (2001). Delay effects on stability. A robust control approach (Springer-Verlag: Heidelberg, LNCIS, vol. 269).

Seyranian, A.P. and Maylibaev, A.A. (2003). Multiparameter stability theory with mechanical applications (World Scientific: Singapore, Series: Stability, Vibration and Control of Systems, vol. 13). 